\title{
Aplicaciones de los Fluidos Supercríticos en la Agroindustria
}

\author{
Reinaldo J. Velasco*, Héctor S. Villada y Jorge E. Carrera
}

Universidad del Cauca, Departamento de Agroindustria, Facultad de Ciencias Agropecuarias, Grupo de Investigación, Aprovechamiento de Subproductos de Origen Agroindustrial (ASUBAGROIN), Calle 5 No. 4-70, Popayán, Cauca-Colombia (e-mail: rvelasco@unicauca.edu.co)

${ }^{*}$ autor a quien debe ser dirigida la correspondencia

\begin{abstract}
Resumen
Esta presenta una revisión sobre el uso del dióxido de carbono supercrítico como solvente para la extracción de compuestos bioactivos presentes en vegetales de actual y potencial uso, en procesos de la agroindustria. Se hace una descripción de la extracción con soxhlet, la cual se usa para evaluar el rendimiento en extracto y en tiempo de extracción, de los métodos tradicionales y recientes de extracción. Se describe la extracción con fluidos supercríticos, se muestran condiciones de operación y se nombran algunos principios bioactivos extraídos de diferentes materias primas vegetales, reportados en la literatura internacional. Para una mejor descripción de las aplicaciones de los fluidos supercríticos en la agroindustria, estas se clasifican en cinco grupos bien diferenciados. Esto permite formarse una idea global clara sobre las posibilidades que ofrecen los fluidos supercríticos para mejorar la competitividad de la agroindustria.
\end{abstract}

Palabras claves: dióxido de carbono, extracción supercrítica, extracción soxhlet, agroindustria

\section{Applications of Supercritical Fluids in the Agroindustry}

\begin{abstract}
A review on the use of supercritical carbon dioxide as a solvent for the bioactive compounds extraction present in vegetables of current and potential use in agroindustrial processes is presented. A description of the extraction with soxhlet, which is used to evaluate the yield in extract and in extraction time of traditional and modern extraction methods is done. Extraction with supercritical fluids is described, operation conditions are showed and bioactive principles extracted from different vegetables raw material reported in the international literature are described. To better described the applications of supercritical fluids in the agroindustry, these are classified in five well defined groups. This allows to get a clear global idea about the possibilities offered by the supercritical fluids to improve the competitiveness of the agroindustry.
\end{abstract}

Keywords: carbon dioxide, supercritical extraction, soxhlet extraction, agroindustry 


\section{INTRODUCCIÓN}

Las plantas poseen una variedad de mezclas de compuestos bioactivos tales como lípidos, grasas, fotoquímicos, fragancias, pigmentos y sabores que son ampliamente utilizados en la agroindustria alimentaria y no alimentaria, en la industria farmacéutica y en la industria cosmética. Para separar estos compuestos (solutos) de la fase sólida, ésta se pone en contacto con una fase líquida, ambas fases entran en contacto íntimo y el (los) soluto(s) se difunde(n) desde el sólido a la fase líquida, lo que permite una separación de los componentes de su estructura natural original. Este proceso se conoce como lixiviación y para realizarlo existen varios métodos. Un proceso importante es la lixiviación de azúcar de las remolachas con agua caliente. Otros procesos muy utilizados consisten en la extracción de aceites vegetales, en los cuales se emplean disolventes orgánicos como hexano, acetona y éter, para extraer aceites de maní, soja, semillas de lino, ricino, girasol o algodón (Geankoplis, 1999).

Los métodos tradicionales de extracción requieren altos tiempos de residencia y grandes cantidades de solvente. Estos métodos se basan en la selección del solvente asociado con el uso de calor y/o agitación e incluyen el soxhlet, la hidrodestilación y maceración mezclada con agua, alcohol o grasa caliente. El soxhlet es una técnica estándar y la principal referencia para evaluar el rendimiento de otros métodos de extracción sólido - líquido (Luque de Castro y García-Ayuso, 1998).

De un tiempo a esta parte se han desarrollado varias técnicas nuevas para la extracción de solutos de matrices sólidas, entre ellas se tiene: la extracción asistida con ultrasonido (Vinatoru, 2001), la extracción asistida con microondas (Kaufmann y Christen, 2002), la extracción con solvente acelerado (Kaufmann y Christen, 2002; Smith, 2002) y la extracción con fluidos supercríticos (Brunner, 2005; Rozzi y Singh, 2002), con el objeto de acortar el tiempo de extracción, disminuir el consumo de solvente, aumentar el rendimiento de extracción y mejorar la calidad del extracto. Se empezará describiendo los métodos de extracción con soxhlet y con fluidos supercríticos, también se citaran algunos resultados comparativos entre estos dos métodos y finalmente se mostrarán algunas de las aplicaciones agroindustriales con fluidos su- percríticos FSC, con el objeto de continuar la divulgación que ya se viene haciendo de éste método y de sus bondades entre los académicos e industriales de nuestros países latinoamericanos.

\section{EXTRACCION CON SOXHLET}

Para la extracción con soxhlet se deben tener en cuenta: la selección del solvente, la matriz sólida y las condiciones de operación.

Selección del solvente: Debe seleccionarse un solvente conveniente de tal forma que ofrezca el mejor balance de varias características deseables: alto límite de saturación y selectividad respecto al soluto por extraer, capacidad para producir el material extraído con una calidad no alterada por el disolvente, estabilidad química en las condiciones del proceso, baja viscosidad, baja presión de vapor, baja toxicidad e inflamabilidad, baja densidad, baja tensión superficial, facilidad y economía de recuperación de la corriente de extracto y bajo costo (Dahlstrom et al., 1999). Cada solvente diferente produce extractos y composiciones específicos (Zarnowski y Suzuki, 2004). El solvente más ampliamente utilizado para extraer aceites comestibles de las plantas es el hexano. El hexano tiene un rango en el punto de ebullición bastante estrecho, de aproximadamente $63-69{ }^{\circ} \mathrm{C}$ y es un excelente solvente de los aceites en lo que se refiere a su solubilidad y facilidad de recuperación. Sin embargo, el n-hexano, el elemento principal del hexano comercial, está ubicado como el número uno en la lista de los 189 contaminantes del aire más riesgosos por la Agencia Americana de Protección del ambiente (Mamidipally y Liu, 2004).

El uso de solventes alternativos tales como: isopropanol, etanol, hidrocarburos, e incluso el agua, se ha incrementado debido a asuntos del medioambiente, la salud, y a preocupaciones de seguridad. Se usó d-cineno y hexano en la extracción de aceite a partir del salvado de arroz y se observó que el d-cineno extrajo una cantidad significativamente superior de aceite que el hexano bajo cualquier serie dada de condiciones (Mamidipally y Liu, 2004). También se ha utilizado agua para extraer el aceite del salvado de arroz a un valor del $\mathrm{pH}$ de 12. El aceite extraído con agua tuvo un volumen más bajo de ácido graso libre y un color más claro que el obtenido con hexano (Hanmoungjai et al., 2000). 
Sin embargo, los solventes alternativos producen a menudo menos recuperación debido a una afinidad molecular disminuida entre el solvente y el soluto. Los costos de los solventes alternativos pueden ser superiores. A veces se agrega un co-solvente para aumentar la polaridad de la fase líquida. Además, se han reportado extracciones de mezclas de isopropanol y el hexano para aumentar el rendimiento y la cinética de extracción ( $\mathrm{Li}$ et al., 2004).

Características de la matriz: La extracción con Soxhlet depende fuertemente de las características de la matriz y de las dimensiones de las partículas puesto que la difusión interna puede ser el paso limitante durante la extracción. Para la extracción total de las grasas de las semillas oleaginosas, se realizó una extracción de 2-h obteniendo un rendimiento del $99 \%$ cuando la dimensión de las partículas era $0.4 \mathrm{~mm}$, mientras que fue necesaria una extracción de 12-h para obtener una eficacia similar si la dimensión de las partículas era $2.0 \mathrm{~mm}$ (Luque-Garcia y Luque de Castro, 2004).

Condiciones de operación: Durante la extracción con Soxhlet, el solvente se recupera normalmente por evaporación. Las temperaturas de extracción y evaporación tienen un efecto significativo en la calidad final de los productos. Además, se ha encontrado que el aceite del salvado de arroz extraído con dcineno era ligeramente más oscuro comparado con el aceite extraído con hexano, probablemente debido a las mayores temperaturas de extracción y evaporación al usar d-cineno como solvente. Las altas temperatura de ebullición para la recuperación del solvente pueden disminuirse usando evaporación flash o separación por membrana para recuperar el solvente (Mamidipally y Liu, 2004).

\section{Comparación Soxhlet y $\mathrm{CO}_{2} \mathrm{SC}$}

La extracción con Soxhlet es una técnica bien establecida. Entre sus ventajas, por encima de otros nuevos métodos como la extracción ayudada con ultrasonido, la ayudada con microondas y la extracción con fluidos supercríticos está la de tener bastantes aplicaciones industriales, buena reproduci-bilidad y eficacia, y menor manipulación del extracto. Sin embargo, comparada con $\mathrm{CO}_{2} \mathrm{SC}$, el método Soxhlet es una técnica anticuada y consumidora de tiempo y de solvente. En la extracción de aceites de rosa silvestre (Rosa canina $L$ ), utilizando $n$-hexano con soxhlet se consumieron 180 minutos para extraer 48.5 $\mathrm{g} / \mathrm{kg}$, mientras que con $\mathrm{CO}_{2} \mathrm{SC}$ a $35^{\circ} \mathrm{C}$ y 250 bar, se consumieron 35 minutos para extraer $57.2 \mathrm{~g} / \mathrm{kg}$, cuando se le agrego propano (cosolvente) al $\mathrm{CO}_{2} \mathrm{SC}$ a $28^{\circ} \mathrm{C}$ y 100 bar se consumieron 35 minutos para obtener 66.8 $\mathrm{g} / \mathrm{kg}$ (Szentmihalyi et al., 2002).

Algunos solventes usados con el Soxhlet convencional se han cuestionado recientemente debido a su toxicidad (n-hexano). El uso de solventes no tóxicos como el $\mathrm{CO}_{2}$ supercrítico y el agua están en el orden del día.

\section{EXTRACCION CON FSC}

Un fluido supercrítico es cualquier substancia a una temperatura y presión por encima de su punto crítico termodinámico. Tiene la propiedad de difundirse a través de los sólidos como un gas, y de disolver los materiales como un líquido. Adicionalmente, puede cambiar rápidamente la densidad con pequeños cambios en la temperatura o presión. Estas propiedades lo hacen conveniente como un sustituto de los solventes orgánicos en los procesos de extracción.

Los fluidos supercríticos (FSC) tienen la capacidad de extraer ciertos compuestos químicos con el uso de determinados solventes específicos bajo la combinación de temperatura y presión (Brunner, 2005; Rozzi y Singh, 2002). En la Tabla 1 se muestran las propiedades críticas de algunos compuestos comúnmente usados como fluidos supercríticos. $\mathrm{El} \mathrm{CO}_{2}$ es el fluido supercrítico más utilizado debido a que es no tóxico, no inflamable, no corrosivo, incoloro, no es costoso, se elimina fácilmente, no deja residuos, sus condiciones críticas son relativamente fáciles de alcanzar y se consigue con diferentes grados de pureza, se puede trabajar a baja temperatura y por tanto, se pueden separar compuestos termolábiles, se puede obtener a partir de procesos de fermentación alcohólica y ayuda a prevenir la degradación térmica de ciertos componentes químicos del alimento cuando son extraídos (Brunner, 2005; Hurtado, 2002; Rosa y Meireles, 2005). El problema con muchos de los fluidos que aparecen en la Tabla 1, en comparación con el $\mathrm{CO}_{2}$ es que hay ciertas dificultades en obtener solventes puros del fluido (Rozzi y Singh, 2002; Wark, 1985). Las 
ventajas de los fluidos supercríticos son: (Bruner, 2005; Hurtado, 2002; Sánchez et al., 2005; Tonthubthimthong et al., 2001; Zkal et al., 2005).

Tabla 1: Propiedades críticas de los FSC

\begin{tabular}{|l|c|c|c|}
\hline \multicolumn{1}{|c|}{ Fluido } & $\begin{array}{c}\mathrm{T}_{\mathrm{c}} \\
\left({ }^{\circ} \mathrm{C}\right)\end{array}$ & $\begin{array}{c}\mathrm{P}_{\mathrm{c}} \\
(\mathrm{bar})\end{array}$ & $\begin{array}{c}\rho \\
\left(\mathrm{Kg} / \mathrm{m}^{3}\right)\end{array}$ \\
\hline Acetileno & 36 & 62.47 & 232.14 \\
\hline Acetona & 235 & 47.00 & 278.00 \\
\hline Agua & 374 & 220.90 & 322.60 \\
\hline Dióxido de Carbón & 31 & 73.86 & 467.60 \\
\hline Etano & 32 & 48.71 & 135.75 \\
\hline Etanol & 240.4 & 61.40 & 276.00 \\
\hline Etileno & 9 & 50.40 & 195.80 \\
\hline Metano & -83 & 45.95 & 161.43 \\
\hline Metanol & 239.4 & 80.90 & 272.00 \\
\hline Propano & 97 & 42.47 & 225.64 \\
\hline Propileno & 91.8 & 46.00 & 232.00 \\
\hline
\end{tabular}

1. Poseen alto coeficiente de difusión y viscosidad más baja que los líquidos; 2. Ausencia de tensión superficial, la cual aumenta la operación de extracción dada la rápida penetración de estos al interior de los poros de la matriz heterogénea; 3 . La selectividad durante la extracción puede ser manipulada dada la variación de las diferentes condiciones de operación temperatura y presión afectando la solubilidad de varios componentes en el fluido supercrítico; 4. La extracción con fluidos supercríticos no deja residuos químicos; 5 . La extracción con $\mathrm{CO}_{2}$ supercrítico permite su fácil recuperación por procesos de reciclaje. EI $\mathrm{CO}_{2}$ supercrítico también ha sido usado en innumerables aplicaciones industriales que incluyen diferentes campos como: alimentos, agricultura, acuicultura, pesticidas, procesos microbianos, petroquímica y farmacéutica (Brunner, 2005; Sánchez et al., 2005; Tonthubthimthong et al., 2001; Vagi et al., 2005).

\section{APLICACIONES AGROINDUSTRIALES}

Algunas aplicaciones comerciales de la extracción con los FSC en la agroindustria agroalimentaria son: el fraccionamiento y la extracción de aceites y grasas, la extracción de antioxidantes naturales, la extracción de alcaloides, aromas y especias. Se describen a continuación estas aplicaciones y los procesos típicos como se llevan a cabo.

\section{Extracción y fraccionamiento de lípidos}

Las aplicaciones de los FSC en esta área se relacionan básicamente con el uso de dióxido de carbono $\left(\mathrm{CO}_{2}\right)$ por las ventajas ya mencionadas y dirigidas hacia la obtención de aceites vegetales a partir de oleaginosas, desacidificación de aceites con alto contenido de ácidos grasos, eliminación de colesterol, aprovechamiento de residuos de la refinación y obtención de compuestos minoritarios de alto valor agregado como son el escualeno, los tocoferoles y los fitosteroles (Hurtado, 2002).

Los aceites vegetales están formados por triglicéridos, diglicéridos, ácidos grasos libres y otros constituyentes minoritarios como tocoferoles y esteroles.

El amplio rango de distribución de sus componentes determina las propiedades físicas del aceite y el uso para el cual es conveniente. El fraccionamiento de aceites resulta de gran interés debido a que permite obtener un aceite o grasa natural ajustado a unas especificaciones muy particulares o una fracción con determinados componentes de interés para fines concretos. Dentro de los posibles procesos de fraccionamiento están la destilación, la extracción con disolventes y la extracción con FSC (Hurtado, 2002). La destilación resulta poco conveniente para usarse con aceites vegetales debido a la presencia de triglicéridos que se rompen a altas temperaturas (Hurtado, 2002).

La extracción con disolventes siempre deja un residuo inherente en el aceite, particularmente cuando la extracción se realiza con hexano que es el disolvente más comúnmente usado, pero la legislación internacional restringe la cantidad de hexano que deben contener los productos extraídos y cada vez será más restrictiva, previéndose que en un futuro se limite su uso y se sustituya por otros disolventes como el $\mathrm{CO}_{2}$ supercrítico.

La extracción con FSC, específicamente con $\mathrm{CO}_{2}$, resulta una alternativa interesante para la extracción y fraccionamiento de aceites vegetales, porque no posee los inconvenientes de los disolventes orgánicos tradicionales, tal como se mencionó anteriormente sobre algunas ventajas que ofrece el uso del $\mathrm{CO}_{2}$ supercrítico al ser no tóxico, ni dejar residuo en sus productos, así como su capacidad selectiva para extraer ciertas sustancias al realizar pequeños cambios de presión y temperatura. Adicionalmente existen estudios que demuestran que el uso de $\mathrm{CO}_{2}$ supercrítico conlleva a disminuir el consumo de 
energía con respecto a procesos de separación convencionales como destilación y lixiviación, entre otros (Tilly et al., 1990).

Sin embargo la ventaja principal de utilizar $\mathrm{CO}_{2}$ supercrítico está en la calidad del aceite obtenido por este medio en comparación con los aceites extraídos con solventes orgánicos tradicionales (Mangold, 1983). Otras ventajas comparativas son: (1) aceites prácticamente libres de fosfolípidos y glicolípidos. Los aceites convencionales contienen de 1 a $3 \%$ de lípidos polares. (2) Menor contenido de hierro. (3) Aceites claros y desodorizados. (4) Menores pérdidas por refinación y menor consumo de soda cáustica (Hurtado, 2002). La desventaja está en la menor estabilidad oxidativa del aceite obtenido con $\mathrm{CO}_{2}$ debido a la ausencia de fosfátidos que en algunos casos protegen al aceite de la autooxidación (List et al., 1985). La extracción de grasas y aceites con FSC cubre un amplio campo en las aplicaciones industriales relacionadas con la obtención misma de estos últimos a partir de oleagino-sas (Esquivel y Gil, 1993).

La obtención de aceites a partir de semillas oleaginosas se ha estudiado desde 1980. Estas investigaciones se han llevado a cabo con $\mathrm{CO}_{2}$ supercrítico a temperaturas entre $40^{\circ} \mathrm{C}$ y $80^{\circ} \mathrm{C}$ y presiones desde 50 hasta 600 bar (Mangold, 1983; List et al., 1984; Penedo y Coelho, 1997). El fraccionamiento de grasas y aceites con $\mathrm{CO}_{2}$ supercrítico, permite obtener productos con funcionalidad mejorada para aplicaciones mas específicas o con mayor valor nutricional (Hurtado, 2002).

El primer proceso comercial publicado para el fraccionamiento de grasas usando FSC fue el proceso Solexol. En esta investigación se utilizó propano supercrítico como disolvente para el fraccionamiento de aceite de pescado, grasa animal y aceites vegetales, permitiendo la construcción de seis plantas con este proceso (Bamberger et al., 1988).

El fraccionamiento de aceite de pescado con $\mathrm{CO}_{2}$ supercrítico también ha sido estudiado. El objetivo fue obtener ácidos grasos poliinsaturados, omega- 3 , a los cuales se les atribuye importantes beneficios para la salud. La destilación a vacío utilizada normalmente en procesos de concentración y separación requiere altas temperaturas que conllevan a alteraciones o descomposiciones de los ácidos grasos (Nilsson et al., 1992).
También se ha estudiado el fraccionamiento de grasa de leche con dióxido de carbono y el contenido de colesterol de tales fracciones (Arul et al., 1987; Arul et al., 1988). Otros investigadores estudiaron el fraccionamiento continuo de grasa de leche en una torre rellena con reflujo. Obtuvieron fracciones de grasa de diferente funcionalidad y menor contenido en colesterol (Lim y Rizvi, 1995).

Otros estudios se han centrado en determinar la solubilidad de ácidos grasos y triglicéridos en dióxido de carbono (Bamberger et al., 1988; Nilsson et al., 1992). También fue estudiada la solubilidad de diferentes ácidos: esteárico y oleico y triglicéridos como tributirina, tripalmitina, triestearina, trioleína y trilinoleína en un intervalo de temperaturas de $40^{\circ} \mathrm{C}$ $-80^{\circ} \mathrm{C}$ y presiones de 80 a 250 bar (Chrastil, 1982). A partir de estos datos de solubilidad por se encontró que a un valor dado de temperatura y presión, los ácidos grasos son más solubles en $\mathrm{CO}_{2}$ que sus correspondientes triglicéridos. Este hecho ha despertado el interés en la desacidificación de aceites, en especial de aceite de oliva, usando $\mathrm{CO}_{2}$ supercrítico. Existen hace varios años patentes y estudios que demuestran la viabilidad de este proceso (Bondioli et al., 1992; Carmelo et al., 1996). Como es sabido, el aceite de oliva de inferior calidad puede contener un alto grado de acidez (4 -15\%), que le suministra un sabor y aroma desagradable. Es poco apto para el consumo humano, por esta razón el aceite de oliva se debe refinar, alterándose su composición nutricional. La extracción con $\mathrm{CO}_{2}$ supercrítico no modifica la calidad nutricional del aceite de oliva virgen procesado (Hurtado, 2002). La Tabla 2, muestra algunos de los productos extraídos (aceite, ácidos grasos y triglicéridos) de materias primas vegetales propuestas por varios trabajos de investigación.

\section{Extracción de Antioxidantes Naturales}

Debido a que el rechazo es cada vez mayor por parte de los consumidores hacia el uso de antioxidantes sintéticos, como por ejemplo el BHA (Butil-Hidroxi-Anisol) y el BHT (ButilHidroxi-Tolueno) y además, dadas las restricciones legales levantadas hacia estos productos, se ha potenciado el empleo de antioxidantes naturales, libres de compuestos químicos sintéticos, como los ácidos fenólicos, los flavonoides y los tocoferoles (Zancan et al., 2002; Yépez et al., 2002). 
Tabla 2: Aceites y lípidos extraídos con $\mathrm{CO}_{2}$ supercrítico

\begin{tabular}{|c|c|c|c|c|}
\hline \multirow[t]{2}{*}{ Materia Prima } & \multirow[t]{2}{*}{ Principio Activo } & \multicolumn{2}{|c|}{$\begin{array}{l}\text { Condiciones de } \\
\text { Extracción }\end{array}$} & \multirow[t]{2}{*}{ Referencias } \\
\hline & & $\mathrm{T}\left({ }^{0} \mathrm{C}\right)$ & $\mathrm{P}(\mathrm{bar})$ & \\
\hline Lúpulo (Humulus lupulus L.) & Ácidos- alfa & $40-60$ & $120-280$ & $\begin{array}{l}\text { Del Valle et al. } \\
\text { (2003) }\end{array}$ \\
\hline Nuez Moscada (Myristica fragans H.) & Aceite & 23 & 90 & Spricigo et al. (2001) \\
\hline Hinojo (Foeniculum vulgare M.) & $\begin{array}{l}\text { Aceite } \\
\text { Triglicéridos }\end{array}$ & $40-50$ & $200-900$ & $\begin{array}{l}\text { Reverchon et al. } \\
(1999)\end{array}$ \\
\hline Avellana & Aceite & $40-60$ & $300-600$ & $\begin{array}{l}\text { Ozkal et al. } \\
\text { (2005) }\end{array}$ \\
\hline Nuez Moscada & Aceite & $50-70$ & $150-300$ & $\begin{array}{l}\text { Rodrigues et al. } \\
(2005)\end{array}$ \\
\hline Hinojo & Triglicéridos & 40 & 300 & $\begin{array}{l}\text { Moura et al. } \\
(2005)\end{array}$ \\
\hline Salvado de Arroz & $\begin{array}{l}\text { Ácidos grasos } \\
\text { libres }\end{array}$ & $50-60$ & $100-400$ & $\begin{array}{l}\text { Danielski et al. } \\
(2005)\end{array}$ \\
\hline Pimienta & Triglicéridos & $35-65$ & $220-500$ & $\begin{array}{l}\text { Del Valle et al. } \\
(2003 a)\end{array}$ \\
\hline Cacao & Triglicéridos & 70 & $200-400$ & Saldaña et al. (2002) \\
\hline Pimienta & Triglicéridos & 40 & $120-200$ & $\begin{array}{l}\text { Del Valle et al. } \\
(2003 b)\end{array}$ \\
\hline
\end{tabular}

Se está utilizando $\mathrm{CO}_{2}$ supercrítico para la obtención de tocoferoles a partir de soja (Del Valle et al., 2005) y subproductos del aceite de oliva (Ibáñez et al., 2000).

También se obtienen antioxidantes naturales a partir de la extracción de plantas tales como salvia y romero, que tienen actividad similar o mayor que las de los antioxidantes sintéticos (Gerard et al., 1995; Señorans et al., 2000). La obtención de Labex, este es un antioxidante obtenido con $\mathrm{CO}_{2}$ a $80-100^{\circ} \mathrm{C}$ y 500 bar, y su posterior fraccionamiento la cual fue lograda en dos partes, una rica en antioxidante y otra en aceite esencial (Nguyen et al., 1994).

En el Centro de Investigación del Cáncer de Heidelberg, en Alemania, se investigó el potencial antioxidante y anticancerígeno de diferentes compuestos fenólicos aislados a partir de aceite de oliva (Owen et al., 2000).

En la Tabla 3, se muestra algunos de los antioxidantes naturales extraídos con $\mathrm{CO}_{2} \mathrm{SC}$.

\section{Extracción de alcaloides, aromas y especias}

Uno de los campos de aplicación de la tecnología con FSC más desarrollados a nivel industrial es la obtención de ingredientes para la agroindustria, perfumes y cosmética.

Otra aplicación clásica de los FSC es la usada en la descafeinación del café. El café contiene del 0.8 - $2 \%$ de cafeína cuyo consumo excesivo puede incidir en la salud de las personas por lo que industrialmente se elimina, pero que por otra parte, tiene un valor agregado por sus a-plicaciones farmacéuticas (Brunner, 2005; Kopcak y Mohamed, 2005; Mohamed et al., 1997).

Otra aplicación interesante es la extracción de compuestos responsables del sabor amargo y característico de la cerveza (humulonas y lupulonas). El $\mathrm{CO}_{2}$ presenta grandes ventajas para la extracción de lúpulo al compararlo con disolventes orgánicos convencionales porque disuelve completamente aceites esenciales, parte de las resinas livianas y pesadas, trazas menores de grasas, ceras, clorofila y sales orgánicas. De este modo los extractos del lúpulo con $\mathrm{CO}_{2}$ permiten conseguir un adecuado balance de aroma y el sabor amargo en la cerveza (Cobos et al., 1997).

La extracción con FSC se ha empleado en la eliminación de nicotina del tabaco (Del Valle et al., 2005). La mayoría de los compuestos responsables de los aromas son solubles $\mathrm{CO}_{2}$

en condiciones supercríticas. Los aspectos tenológicos de la producción de aceites, colorantes, aromas, esencias, saborizantes, edulcorantes, han sido revisados por otros investigadores (Starmans y Nijhuis, 1996; Del Valle y Aguilera, 1999; Del Valle et al., 2005). Una lista de productos extraídos de materias 
primas vegetales en diferentes trabajos de investigación se indica en la Tabla 4 . En la actualidad existen plantas comerciales de extracción con FSC [Aromtech Ltd., Finland (www.aromtech.com); Flavex Naturextrakte $\mathrm{GmbH}$, Germany (www.flavex.com); Florys SpA, Italy (www.officinalidisardegna.it/florys), Raps GmbH\&Co. Germany (www.raps.com), Super Critical Extraction in New Zealand (Scenz) Ltda (www.supercritical.co.nz)]. Son plantas que poseen extractores de 100-500 L, operan bajo procesos discontinuos o semicontinuos y trabajan con diferentes materias primas (Del Valle et al, 2005).

\section{Uso en técnicas analíticas}

El $\mathrm{CO}_{2} \mathrm{SC}$ se ha usado en algunos métodos analíticos como detector del contenido de grasa por medio de la fase móvil usando técnicas analíticas de cromatografía (Rozzi y Singh, 2002). En esta sección se habla de la detección del contenido de grasas por FSC y el uso de la técnica FSC. El uso de métodos analíticos por medio de los FSC se ha aplicado a una gran variedad de productos alimenticios desde la carne de res hasta las semillas y vegetales que presentan un alto contenido de ácidos grasos. Se ha demostrado que el uso de técnicas analíticas con apoyo de FSC presenta mayores ventajas que las técnicas tradicionales. El análisis del contenido de aceites en semillas de soya, girasol, algodón, colza, entre otros materiales biológicos, ha presentado mayor eficiencia en la extracción que la obtenida por métodos convencionales (Taylor et al., 1997; Taylor y King, 2000).
Se demostró la similitud entre el método de extracción de Röse-Gottlieb comparado con el FSC; pero la rapidez en la extracción marcó la diferencia en el FSC y el tipo de solvente orgánico utilizado en la extracción (Dionisi et al., 1999). El cambio en peso de muestras durante este proceso con FSC fue detectado por medio del uso de un detector Piezoeléctrico. Esta técnica permitió determinar la pérdida de peso en la muestra después de extraer totalmente la grasa. Además, es una determinación rápida y segura que las técnicas tradicionales (Manganiello et al., 2000).

El $\mathrm{CO}_{2} \mathrm{SC}$ también se ha utilizado como fase móvil en cromatografía de fluido supercrítico desde 1980. El uso de FSC como fase móvil para cromatografía provee de alguna ventajas analíticas sobre las técnicas convencionales GC o HPLC. El poder de los FSC como solvente puede ser manipulado por los cambios de temperatura y/o presión (densidad) de la fase móvil (Poole, 2000). En el caso del $\mathrm{CO}_{2}$, cuando la densidad es baja sus condiciones de operación son similares a las del hexano y cuando la densidad es alta sus condiciones son similares a las del diclorometano. La composición de la fase móvil puede ser variada durante la separación de compuestos por HPLC, la densidad de la fase móvil se puede variar durante el proceso de separación por cromatografía con FSC. Y se pueden adicionar co-solventes polares a la fase móvil, los cuales ayudan a separar compuestos polares que no se separan fácilmente con $\mathrm{CO}_{2}$ y aumenta el rendimiento de la extracción con FSC (Brunner, 1994).

Tabla 3: Antioxidantes naturales extraídos con $\mathrm{CO}_{2}$ supercrítico

\begin{tabular}{|c|c|c|c|c|}
\hline \multirow[t]{2}{*}{ Materia Prima } & \multirow[t]{2}{*}{$\begin{array}{l}\text { Principio } \\
\text { Activo }\end{array}$} & \multicolumn{2}{|c|}{$\begin{array}{l}\text { Condiciones de } \\
\text { Extracción }\end{array}$} & \multirow[t]{2}{*}{ Referencias } \\
\hline & & $\mathrm{T}\left({ }^{0} \mathrm{C}\right)$ & $\mathrm{P}$ (bar) & \\
\hline Cascara de pistacho (Pistachia vera) & Antioxidante & $35-60$ & $\begin{array}{l}101- \\
355\end{array}$ & Goli et al. (2005) \\
\hline $\begin{array}{l}\text { Rizomas de Ginger (Zingiber officinale } \\
\text { R.) }\end{array}$ & Gingerol & $20-40$ & $\begin{array}{l}150- \\
200\end{array}$ & $\begin{array}{l}\text { Martínez et al. } \\
(2003)\end{array}$ \\
\hline $\begin{array}{l}\text { Rizomas de Ginger (Zingiber officinale } \\
\text { R.) }\end{array}$ & Gingerol & $20-40$ & $\begin{array}{l}100- \\
300\end{array}$ & $\begin{array}{l}\text { Rodrígues et al. } \\
(2002)\end{array}$ \\
\hline $\begin{array}{l}\text { Rizomas de Ginger (Zingiber officinale } \\
\text { R.) }\end{array}$ & Gingerol & $20-35$ & $\begin{array}{l}200- \\
250\end{array}$ & Zancan et al. (2002) \\
\hline Romero (Rosmarinus officinalis L.) & Antioxidante & $40-60$ & $\begin{array}{l}100- \\
400\end{array}$ & Ramírez et al. (2004) \\
\hline Semillas de Cilantro (Coriander sativum) & Antioxidante & $58-85$ & $\begin{array}{l}116- \\
280\end{array}$ & Yepez et al. (2002) \\
\hline Yerba Buena (Hierochloe odorata) & Antioxidante & 40 & $\begin{array}{c}250- \\
350\end{array}$ & Grigonis et al. (2005) \\
\hline
\end{tabular}


Tabla 4: Alcaloides, aromas y especias extraídos con $\mathrm{CO}_{2}$ supercrítico

\begin{tabular}{|c|c|c|c|c|}
\hline \multirow[t]{2}{*}{ Materia Prima } & \multirow[t]{2}{*}{$\begin{array}{l}\text { Principio } \\
\text { Activo }\end{array}$} & \multicolumn{2}{|c|}{$\begin{array}{l}\text { Condiciones de } \\
\text { Extracción }\end{array}$} & \multirow[t]{2}{*}{ Referencias } \\
\hline & & $\mathrm{T}\left({ }^{0} \mathrm{C}\right)$ & $\mathrm{P}$ (bar) & \\
\hline Anís (Pimpinella anisum L.) & Aceite esencial & 30 & $80-180$ & Rodrígues et al. (2003) \\
\hline Bulbo de Clavo (Eugenia caryophillus) & Aceite esencial & 50 & $90-120$ & Ruetsch et al. (2003) \\
\hline Bulbo de Clavo (Eugenia caryophillus) & Aceite esencial & $\begin{array}{l}10- \\
35\end{array}$ & $66-100$ & Rodrígues et al. (2002) \\
\hline Eucalipto (Eucalyptus tereticornis) & Aceite esencial & $\begin{array}{l}10- \\
25\end{array}$ & $66.7-78.5$ & Rodrígues et al. (2002) \\
\hline Manzanilla (Chamomilla recutita L.) & Aceite esencial & $\begin{array}{l}30- \\
40\end{array}$ & $100-200$ & Povh et al. (2001) \\
\hline Pimienta (Lippia sidoides C.) & Aceite esencial & $\begin{array}{l}10- \\
25\end{array}$ & $66.7-88.5$ & Sousa et al. (2002) \\
\hline Pimienta Negra (Piper nigrum L.) & Aceite esencial & $\begin{array}{c}30- \\
50\end{array}$ & $150-300$ & Ferreira et al. (2002) \\
\hline Romero (Rosmarinus officinalis L.) & Aceite esencial & $\begin{array}{c}38- \\
48\end{array}$ & $100-160$ & Coelho et al. (1997) \\
\hline Tomillo (Thymus vulgaris) & Aceite esencial & 40 & 200 & $\begin{array}{l}\text { Viera de Melo et al. } \\
(2000)\end{array}$ \\
\hline $\begin{array}{l}\text { Semillas de Guaraná (Paullinia cupana } \\
\text { M.) }\end{array}$ & Alcaloide & $\begin{array}{l}40- \\
70\end{array}$ & $100-400$ & Saldaña et al. (2002a) \\
\hline Palma (Lat. palum) & Alcaloide & $\begin{array}{c}40- \\
80\end{array}$ & $207-483$ & Zaidul et al. (2006) \\
\hline Pimienta (Capsicum annuum L.) & Capsaicinoides & 40 & $120-320$ & Del Valle et al. (2003) \\
\hline Cúrcuma (Curcuma longa L.) & Colorante & 45 & $250-300$ & $\begin{array}{l}\text { Chassagnez-Méndez } \\
\text { et al. (2000) }\end{array}$ \\
\hline Stevia (Stevia rebaudiana B.) & Glúcidos & 30 & $200-250$ & Yoda et al. (2003) \\
\hline Curcuma (Curcuma longa $L$ ) & Oleoresina & 45 & $200-300$ & $\begin{array}{l}\text { Chassagnez et al. } \\
\text { (1997) }\end{array}$ \\
\hline Caléndula & Aceite & $20-40$ & $120-200$ & Campos et al. (2005) \\
\hline Cebolla Cabezona & Aceite & $37-50$ & $207-287$ & $\begin{array}{l}\text { Saengcharoenrat y } \\
\text { Guyer (2004) }\end{array}$ \\
\hline Corcho (Quercus suber L) & $\begin{array}{l}\text { Triterpenos } \\
\text { Esteroides }\end{array}$ & 50 & 220 & Castola et al. (2005) \\
\hline
\end{tabular}

\section{Otras extracciones y aplicaciones}

Las aplicaciones de los FSC (especialmente del $\mathrm{CO}_{2}$ supercrítico), son muy amplias, por ello es de resaltar otros campos de aplicación también de interés industrial como son la eliminación de aceite en papas y croquetas de yuca fritas (Hurtado, 2002); la eliminación de alcohol en bebidas alcohólicas (Señorans et al., 2001); la extracción de aromas y sabores de jugos cítricos (Temelli et al., 1998); también se han desarrollado nuevos procesos de extrusión y esponjado con $\mathrm{CO}_{2} \mathrm{SC}$ (Alavi et al., 2003, 2003a; Gogoi et al, 2000; Jeong y Toledo, 2004). Se han inactivado bacterias y esporas por $\mathrm{CO}_{2} \mathrm{SC}$ (Dillow et al., 1999; Shimoda et al., 2002; Watanabe et al., 2003). Entre otras aplicaciones se puede mencionar el fraccionamiento de grasas y aceites, la eliminación de ácidos grasos libres (aceite de oliva) así como de otros aceites, la desodorización y la extracción de aceite a partir de lecitina y el aprovechamiento de residuos obtenidos de los procesos de refinación (Esquivel y Gil, 1993).

Como se observa el panorama de aplicación de los FSC es muy prometedor, más en Latinoamérica y específicamente en Colombia, donde la biodiversidad es tan grande y existe una innumerable cantidad de especies que pueden ser aprovechadas para obtener productos de alto valor agregado. Algunos investigadores colombianos han publicado resultados de trabajos sobre extracciones de plantas nativas con FSC (Stashenko et al., 2004). En este sentido extraer los compuestos bioactivos, como aromas y sabores, entre muchos 
otros, a partir de plantas autóctonas, puede ser un campo de aplicación importante desde el punto de vista investigativo y económico para varios de nuestros países de LatinoAmérica (Del Valle et al., 2005).

\section{CONCLUSIONES}

Cada vez se conocen mejor las propiedades de los fluidos supercríticos que son útiles para los diferentes campos de aplicación de la agroindustria alimentaria. Permanentemente se están produciendo nuevas investigaciones en este campo y con ejemplos exitosos que pueden convertirse en agroindustrias para la región Latinoamericana. Esta revisión busca despertar el interés en nuestras poblaciones para su utilización en los desarrollos de nuevos producto y procesos a partir de materias primas autóctonas.

\section{REFERENCIAS}

Alavi, S. H., S. S. H. Rizvi. y P. Harriott, Process dynamics of starch-based microcellular foams produced by supercritical fluid extrusion, l: model development, Food Research Intern., 36 (4), 309-319 (2003).

Alavi, S. H., S. S. H. Rizvi. y P. Harriott, Process dynamics of starch-based microcellular foams produced by supercritical fluid extrusion. II: Numerical simulation and experimental evaluation, Food Research International, 36 (4), 321-330 (2003a).

Arul, J., A. Boudreau., J. Makhlouf., R. Tardif. y B. Grenier, Distribution of cholesterol in milk fat fractions, J. Dairy Res., 55, 361-371 (1988)

Arul, J., A. Boudreau., J. Makhlouf., R. Tardif. y M. R. Sahasrabudhe, Fractionation of anhydrous milk fat by superficial carbon dioxide, J. Food Sci., 52(5), 1231-1236 (1987).

Bamberger, T., J. C. Erickson. y C. L. Cooney, Measurement and model prediction of solubilities of pure fatty acids, pure triglycerides and mixtures of triglycerides in $\mathrm{CO}_{2} \mathrm{SC}$, J. Chem. Eng. Data, 33, 327-333 (1988).

Bondioli, P., Mariani, C., Fedell, E., Mossa, A., Muller. y A. Lampante, Olive oil refining with supercritical carbon dioxide, J. Am. Oil Chem. Soc., 69 (5), 477-480 (1992).

Brunner, G, Supercritical fluids: technology and application to food processing, Journal of Food Engineering, 67. 21-33 (2005).
Brunner, G, Gas Extraction An Introduction to Fundamentals of Supercritical Fluids and the Application to the Separation Processes, Springer, New York, USA (1994).

Campos, M. A. S., M. Z. Michielin., L Danielski. y R. S. Ferreira, Experimental data and modeling the supercritical fluid extraction of marigold (Calendula officinalis) oleoresin, J.Supercrit. Fluids, 34 (2), 163-170 (2005)

Carmelo, P. J., P. J. Pereira., P. C. Simoes., y M. Nunes da Ponte, Scaleup of a supercritical extraction unit for the deacidification of olive oil, High Press. Chem. Eng., 12, 487-492 (1996).

Castola, V., B. Manrongiu., A. Bighelli., C. Floris., A. Laï. y J. Cassanova, Extractives of cork (Quecus suber L.): chemical composition of dicloromethane and supercritical $\mathrm{CO}_{2} \mathrm{ex}$ tract. Industrial Crops and products, 21, 6569 (2005).

Chassagnez-Mendez, A. L., N. T. Machado., M. E. Araujo.,J. G. Maia. y M. A. M. Meireles, Supercritical $\mathrm{CO}_{2}$ extraction of curmumins and essential oil from the rhizomes of turmeric (Curcuma longa L.), Industrial \& Engineering Chemistry Research, 39, 4729-4733 (2000).

Chassagnez, A. L. M., C. F. N. Correa y A. A. M. Meireles, "Tração de oleoresina de cúrcuma (Curcuma longa L) com $\mathrm{CO}_{2}$ supercrítico. Ciência e Tecnologia de Alimentos, ISSN: 0101-2061 (en línea), 17 (4), 1997. (fecha de consulta: Abril 10, 2006), acceso libre, http://www.scielo.br/scielo.php.

Chrastil, J, Solubility of solids and liquids in supercritical gases, J. Phys. Chem., 86, 30163021(1982).

Cobos. A., Díaz. O., L. Perales. y J. A. Ordóñez, El dióxido de carbono supercrítico en la elaboración de alimentos de origen vegetal. Otras aplicaciones, Alimentación, equipos y tecnología, 16 (8), 55-63 (1997).

Coelho, L. A. F., J. V. Oliveira., S. G. d’Avila., J. H. Y. Villegas. y F. M. Lanças, SFE of rosemary oil: assessment of the influence of process variables and extract characterization, Journal of High Resolution Chromatography, 20, 431- 436 (1997).

Danielski, L., C. Zetzl., H. Hense. y G. Brunner, A process line for the production of raffinated rice oil from rice bran, J. Supercrit. Fluids, 34 (2), 133-141 (2005). 
Dahlstrom, D. A. y otros catorce autores, "Liquid-Solid Operations and Equipment" In Perry's Chemical Engineers' Handbook, by Perry, R. H., D. W. Green. y J. O. Maloney, $7^{\text {th }}$ edition, McGraw-Hill, section 18, pp 58-59 (1999).

Del Valle, J. M., J. C. De La Fuente. y A. C. Damian, Contributions to supercritical extraction of vegetable substrates in Latin America, J. Food Eng., 67, 35-57 (2005).

Del Valle, J. M., O. Rivera., O. Teuber. y M. T.Palma, Supercritical $\mathrm{CO}_{2}$ extraction of chilean hop (Humulus lupulus) ecotypes, J. Sci. Food Agric., 83, 1349-1356 (2003).

Del Valle, J. M., M. Jimenez. y J. C. de la Fuente, Extraction kinetics of pre-pelletized Jalapeño peppers with supercritical $\mathrm{CO}_{2}, \mathrm{~J}$. Supercrit. Fluids, 25, 33-44 (2003a).

Del Valle, J. M., M. Jimenez., P. Napolitano., C. Zetzl, C. y G. Brunner, Supercritical carbon dioxide extraction of pelletized Jalapeño peppers, J. Sci. Food Agric., 83, 550-556 (2003b).

Del Valle, J. M. y J. M. Aguilera, Extracción con $\mathrm{CO}_{2}$ a alta presión fundamentos y aplicaciones en la industria de alimentos, Food Sci. Technol. Int. , 5 (1), 1-24 (1999).

Dillow, K. A., Dehghani, F., S. J. Hrkach., R. N. Foster. y R. Langer, Bacterial inactivation by using near and supercritical carbon dioxide, Medical Sci., 96 (18), 10344 - 10348 (1999).

Dionisi, F., B. Hug., J. M. Aeschlimann. y A. Houllemar, Supercritical $\mathrm{CO}_{2}$ extraction for total fat analysis of food products, J. Food Sci. 64(4), 612-615 (1999).

Esquivel, M. y M. G. Bernardo-Gil, El uso de fluidos supercríticos en la industria de aceites alimentarios, Grasas y Aceites, 44 (1), 1-5 (1993).

Ferreira, S. R. S. y M. A. A. Meireles, Modeling the supercritical fluid extraction of black pepper (Piper nigrum L.) essential oil, J. Food Eng., 54, 263-269 (2002).

Geankoplis, Ch. J., "Procesos de Transporte y Operaciones Unitarias", $3^{a}$ edición, 800-801. Editorial Continental, S. A de C. V. México, México (1999).
Gerard, D., K. W. Quirin. y E. Schwarz, $\mathrm{CO}_{2^{-}}$ extracts from rosemary and sage, Food Mark. and Technol., 9 (5), 46-55 (1995).

Gogoi, B. K., S. H. Alavi. y S. S. H. Rizvi, Mechanical properties of protein-stabilized starch-based supercritical fluids extrudates, Int. J. Food Properties, 3 (1), 37-58 (2000).

Goli, A. H., M. Barzegar y M. A. Sahara, Antioxidant activity and total phenolic compounds of pistachio (Pistachia vera) hull extracts, Food Chemistry, 92, 521-525 (2005).

Grigonis, D., P. R. Venskutonis., B. Sivik., M. Sandahl. y C. S. Eskilsson, Comparison of different extraction techniques for isolation of antioxidants from sweet grass (Hierochloe odorata), J. Supercrit. Fluids, 33, 223-233 (2005).

Hanmoungjai, P., L. Pyle, y K. Niranjan, Extraction of rice bran oil using aqueous media, J. Chem. Technol. Biotechnol., 75, 348-352. (2000).

Hurtado, B. A. M, Estudio del proceso de extracción de componentes minoritarios de aceite de oliva con $\mathrm{CO}_{2}$ supercrítico en contracorriente, Tesis Doctoral. Universidad Autónoma de Madrid, Dpto. de Ing. Química, Madrid, España (2002).

Ibáñez, E., J. Palacios., F. J. Señorans., G. Santa-Maria. y G. Reglero, Isolation and separation of tocopherols from olive by products with supercritical fluids, J. Am. Oil Chem. Soc., 77 (2), 187-190 (2000).

Jeong, H. S. y R. T. Toledo, Twin-screw extrusion at low temperature with carbon dioxide injection to assist expansion: extrudate characteristics, J. Food Eng., 63, 425432 (2004).

Kaufmann, B. y P. Christen, Recent extraction techniques for natural products: Microwaveassisted extraction and pressurized solvent extraction, Phytochem. Anal., 13, 105-113 (2002).

Kopcak, U. y R. S. Mohamed, Caffeine solubility in supercritical carbon dioxide/cosolvent, J. Supercrit. Fluids, 34 (2), 209-214 (2005).

Li, H., L. Pordesimo, L., y J. Weiss, High intensity ultrasoundassisted extraction of oil 
from soybeans, Food Research International, 37, 731-738. (2004).

Lim, S. y S. S. H. Rizvi, Continuous supercritical fluid processing of anhydrous milk fat in a packed column, J. Food Sci., 60, 889-893 (1995).

List, G. R., J. P. Friedrich y J. Pominski, Processing characteristics and oxidative stability of soybean oil extracted with supercritical carbon dioxide at $50^{\circ} \mathrm{C}$ and $800 \mathrm{psi}$, J. Am. Oil Chem. Soc., 62, 82-84 (1985).

List, G. R., J. P. Friedrich y J. Pominski, Characterization and processing of cottonseed oil obtained by extraction with supercritical carbon dioxide, J. Am. Oil Chem. Soc., 61, 1847-1849 (1984).

Luque de Castro, M. D. y L. E. Garcia-Ayuso, Soxhlet extraction of solid materials: An outdated technique with a promising innovative future, Anal. Chim. Acta, 369, 1-10. (1998).

Luque-Garcia, J. L. y M. D. Luque de Castro, Ultrasoundassisted Soxhlet extraction: An expeditive approach for solid sample treatment-Application to the extraction of total fat from oleaginous seeds, J. Chromatogr. A, 1034, 237-242. (2004).

Mamidipally, P. K. y S. X. Liu, First approach on rice bran oil extraction using limonene, Eur. J. Lipid Sci. Technol., 106, 122-125. (2004).

Manganiello, L., A. Rios. y M. Valcarcel, Automatic microgravimetric determination of fats in milk products by use of supercritical fluid extraction with on-line piezoelectric detection, J. Chromatogr. A, 874, 265-274 (2000).

Mangold, H K, Liquified gases and supercritical fluids in oilseed extraction, J. Am. Oil Chem. Soc., 60, 178A-179A (1983).

Martínez, J., A. R. Monteiro., P. T. Rosa., M. O. M. Marques y M. A. A. Meireles, Multicomponent model to describe extraction of ginger oleoresin with supercritical carbon dioxide, Ind. Eng. Chem. Res., 42, 1057-1063 (2003).

Mohamed, R. S., D. M. A. Saldaña. y P. Mazzafera, Extração dos Alcalóides: Cafeína e Trigonelina dos Grãos de Café $\mathrm{Com} \mathrm{CO}_{2}$ Supercrítico, Ciência e Tecnología de Alimentos, 17 (4), 371-376 (1997).
Moura, L. S., R. N. Carvalho., M. B. Stefanini., L. C. Ming. y M. A. Meireles, Supercritical fluid extraction from fennel (Foeniculum vulgare): global yield, composition and kinetic data, J. Supercrit. Fluids, 35 (3), 212-219 (2005).

Nguyen, U., D. A. Evans. y G. Frakman, Natural antioxidants produced by supercritical extraction. Supercritical Fluid Processing of Food and Biomaterials, Ed, by Rizvi, S. S. H. Blackie academic and professional, New York, USA (1994).

Nilsson, W. B., G. T. Seaborn. y J. K. Hudson, Partition coefficients for fatty acid esters in supercritical fluid $\mathrm{CO}_{2}$ with and without ethanol, J. Am. Oil Chem. Soc., 69, 305-308 (1992).

Owen, R. W., A. Giacosa., W. E. Hull., R. Haubner. y B. Spriegelhalder, The antioxidant lanticancer potential of phenolic compounds isolated from olive oil, Eur. J. Cancer, 36 (10), 1235-1247 (2000).

Ozkal, S. G., U. Salgın. y M. E. Yener, Supercritical carbon dioxide extraction of hazelnut oil. J. Food Eng., 69 217-223 (2005).

Penedo, P. L. M. y G. L. V. Coelho. "Purificação de óleos vegetais por extração com $\mathrm{CO}_{2}$ supercrítico", Rev Scielo Brasil ISSN 0101-2061 (en línea), 17 (4), 1997 (fecha de consulta: Abril 10, 2006), acceso libre, http:// www.scielo.br/scielo.php

Poole, C. F, Progress in packed column supercritical fluid chromatography: materials and methods, J. Biochem. Bioph. Methods, 43, 3-23 (2000)

Povh, N. P., M. O. M. Marques. y M. A. A. Meireles, Supercritical $\mathrm{CO}_{2}$ extraction of essential oil and oleoresin from chamomile (Chamomilla recutita [L.] Rauschert), J. Supercrit. Fluids, 21, 245-256 (2001).

Ramírez, P., F, J. Senoráns., E. Ibañez. y G. Reglero, Separation of rosemary antioxidant compounds by supercritical fluid chromatography on coated packed capillary columns, J. Chromatogr. A, 1057, 241-245, (2004).

Reverchon, E., J. Daghero., C. Marrone., M. Mattea. y M. Poletto, Supercritical fractional extraction of fennel seed oil and essential oil: experiments and mathematical modeling, Ind. Eng. Chem. Res, 38, 3069-3075 (1999). 
Rodrigues, J. E., M. E. Araújo., F. F. M. Azevedo. y N. T. Machado, $P$ ozkal hase equilibrium measurements of Brazil nut (Vertholletia excelsa) oil in supercritical carbon dioxide, J. Supercrit. Fluids, 34 (2), 223-229 (2005).

Rodrigues, M. R. A., E. B. Carabao., J. G. Dos Santos., C. Dariva. y J. V. Oliveira, The effects of temperature and pressure on the characteristics of the extracts from highpressure $\mathrm{CO}_{2}$ extraction of Majorana hortensis Moench, J. Agric. Food. Chem, 51, 453-456 (2003).

Rodrigues, V. M., E. M. B. D. Sousa., A. R. Monteiro., O. Chiavone-Filho., M. O. M. Marques. y M. A. A. Meireles, Determination of the solubility of extracts from vegetable raw material in pressurized $\mathrm{CO}_{2}$ : a pseudo-ternary mixture formed by cellulosic structure + solute + solvent, J. Supercrit. Fluids, 22, 21-36 (2002).

Rosa, P. T. V. y M. A. A. Meireles, Rapid estimation of the manufacturing cost of extracts obtained by supercritical fluid extraction, J. Food Eng, 67, 235-240 (2005).

Rozzi, N. L. y R. K. Singh, Supercritical Fluids and the Food Industr, Comprehensive Reviews in Food Science and Food Safety, 1, 33-44 (2002).

Ruetsch, L., J. Daghero. y M. Mattea, Supercritical extraction of solid matrices. Model formulation and experiments. Latin American Applied Research, 33, 103-107 (2003).

Saengcharoenrat, C. y D. E. Guyer, Effects of supercritical carbon dioxide conditions on onion oil desorption, J. Food Eng, 63, 33-37 (2004).

Saldaña, M. D. A., R. S. Mohamed. y P. Mazzafera, Extraction of cocoa butter from Brazilean cocoa beans using supercritical $\mathrm{CO}_{2}$ and ethane, Fluid Phase Equilibria, 194-197, 885-894 (2002).

Saldaña, M. D. A., C. Zetzl., R. S. Mohamed. y G. Brunner, Extraction of methylxanthines from guarana seeds, mate leaves, and cocoa beans using supercritical carbon dioxide and ethanol, J. Agric. Food. Chem, 50, 4820-4826 (2002a).

Sánchez, M. D. M., C. Mantell., M. Rodríguez., E. Martínez de la Ossa., L. M. Lubian. y
O. Montero, Supercritical fluid extraction of carotenoids and chlorophylla from Nannochloropsis gaditana, J. Food Eng, 66. 245-251 (2005).

Señorans, F. J., E. Ibáñez., S. Cavero., J. Tabera. y G. Reglero, Liquid chromatogramphicmass spectrometric analysis of supercritical fluid extracts of rosemary plant, J. Chromatogr. A, 870,491-499 (2000).

Señorans, F. J., A. Ruiz., E. Ibáñez., J. Tabera. y G. Reglero, Optimization of countercurrent supercritical fluid extraction conditions for spirits fractionation, J. Supercrit. Fluids, 21, 41-49 (2001).

Shimoda, M., H. Kago., N. Kojima., M. Miyake., Y. Osajima. y I. Hayakawa, Accelerated Death Kinetics of Aspergillus niger Spores under High-Pressure Carbonation, Appl. Envir. Microbiol, 68 (8), 4162- 4167 (2002).

Smith, R. M, Extractions with superheated water, J. Chromatogr. A, 975, 31-46 (2002).

Sousa, E. M. B. D., O. Chiavone-Filho., M. T. Moreno., D. N. Silva., M. O. M. Marques. y M. A. A. Meireles, Experimental results for the extraction of essential oil from lippia sidoides Cham using pressurized carbon dioxide, Brazilian J. Chem. Eng, 19, 229-241 (2002).

Spricigo, C. B., A. Bolzan. y L. T. Pinto, Mathematical modeling of nutmeg essential oil extraction by liquid carbon dioxide, Latin American Applied Research, 31, 397-401 (2001).

Stashenko, E. E., B. E. Jaramillo. y J. R. Martinez, Comparison of different extraction methods for the analysis of volatile secondary metabolites of Lippia alba (Mill.) N.E. Brown, grown in Colombia, and evaluation of its in vitro antioxidant activity, J. Chromatogr. A, 1025, 93-103 (2004).

Starmans, A. J. y H. H. Nijhuis, Extraction of secondary metabolites from plant material: A review, Trends Food Sci. Tech, 7, 191-197 (1996)

Szentmihalyi, K., P. Vinkler., B. Lakatos., V. Illes. y M. Then, Rose hip (Rosa canina L) oil obtained from waste hip seeds by different extraction methods. Bioresource Technology, 82, 195-201 (2002).

Información Tecnológica - Vol. 18 No 1 - 2007 
Taylor, S. L. y J. W. King, Optimization of the extraction and fractionation of corn bran oil using analytical supercritical fluid chromatogramphy, J. Chromatogr. Sci, 38, 91-94 (2000).

Taylor, S. L., F. J. Eller. y J. W. King, A comparison of oil and fat content in oilseeds and ground beef using supercritical fluid extraction and related analytical techniques, Food Res Int., 30 (5), 365-370 (1997).

Temelli, F., C. S. Chen. y R. J. Braddock, Supercritical fluid extraction in citrus oil processing, Food Technol., 42 (6), 145-150 (1998).

Tilly, K. D., R. P. Chaplin y N. R. Foster, Supercritical fluid extraction of the triglycerides present in vegetable oils, Separ. Sci. Technol, 25.(4),357-367(1990).

Tonthubthimthong, P., S. Chuaprasert., P, Douglas. y W, Luewisutthicha, Supercritical $\mathrm{CO}_{2}$ extraction of nimbin from neem seeds an experimental study. J. Food Eng, 47, 289-293 (2001).

Vagi, E., B. Simandi., A. Suhajda. y E. Hethelyi, Essential oil composition and antimicrobial activity of Origanum majorana L. extracts obtained with ethyl alcohol and supercritical carbon dioxide, Food Research International, 38. 51-57 (2005).

Viera de Melo, S. A. B., G. M. N. Costa., A. Casula. y B. Pittau, Supercritical $\mathrm{CO}_{2}$ extraction of essential oils from Thymus vulgaris, Brazilian J. Chem. Eng, 17, 367-371 (2000).

Vinatoru, M, An overview of the ultrasonically assisted extraction of bioactive principles from herbs, Ultrasonics Sonochemistry, 8, 303-313 (2001).

Wark, K. "Termodinámica". 4ª edición, 792. McGraw-Hill Inc., México, D. F., México (1985).
Watanabe, T., S. Furukawa., J. Hirata., T. Koyama., H. Ogihara. y M. Yamasaki, Inactivation of Geobacillus stearothermophilus Spores by High-Pressure Carbon Dioxide, Treatment. Appl. Envir. Microbiol., 69(12); 7124 - 7129 (2003).

Yépez, B., M. Espinosa., S. López. y G. Bolanos, Producing antioxidant fractions from herbaceous matrices by supercritical fluid extraction, Fluid Phase Equilibria, 194, 879 884 (2002).

Yoda, S. K., M. O. M. Marques., A. J. Petenate. y M. A. A. Meireles, Supercritical fluid extraction from Stevia rebaudiana Bertoni using $\mathrm{CO}_{2}$ and $\mathrm{CO}_{2}$ plus water: extraction kinetics and identification of extracted components, J. Food Eng, 57, 125-134 (2003).

Zaidul, I. S. M., N. A. N. Norulaini., A. K. O. Mohd. y R. L. J. Smith, Supercritical carbon dioxide $\left(\mathrm{SC}-\mathrm{CO}_{2}\right)$ extraction and fractionation of palm kernel oil from palm kernel as cocoa butter replacers blend, J. Food Eng, 73, 210216 (2006)

Zancan, K. C., M. O. M. Marques., A. J. Petenate. y M. A. A. Meireles, Extraction of ginger (Zingiber officinale Roscoe) oleoresin with $\mathrm{CO}_{2}$ and co-solvents: a study of the antioxidant action of the extracts, J. Supercrit Fluids, 24, 57-76 (2002).

Zarnowski, R. y Y. Suzuki, Expedient Soxhlet extraction of resorcinolic lipids from wheat grains, J Food Composit. Anal, 17, 649-664. (2004)

Zkal, S. G. O., U. Salgın. y M. E. Yener, Supercritical carbon dioxide extraction of hazelnut oil, J. Food Eng, 69, 217-223 (2005). 
Septiembre 2007

\section{Congreso Argentino de Ingeniería Rural}

$$
19 \text { al } 22 \text { de Septiembre de } 2007
$$$$
\text { Córdoba - Argentina }
$$

Universidad Nacional de Córdoba cadir007@agro.uncoredu_http//www.agricultura deprecision.org/eventos/2007/20070919cadir.htm

\section{Octubre 2007}

\section{Encuentro Latinoamericano de Biotecnologia} Agropecuaria, REDBIO 2007- Chile

\section{1 de 5 de Octubre de 2007} Viña del Mar - Chile

Sr. Carlos Fernandez, profesional también de FIA carlos.fernandez@fia.gob.cl http $/ /$ www.redbio.org/newsredbio. asp?id=294

\section{Conferencia IFAC sobre Automatización Eficiente}

en el Desarrollo y Manufactura de Productos

2 al 5 de Octubre de 2007

Monte Rey - Nuevo León - México

Arturo Molina Gutierrez, ITESM, Monterrey, México armolina@itesm.mx, heinz.erbe@tu-berlin.de http://cidyt.mty.itesm.mx/ifac-CEA07/index.html

\section{Congreso Internacional de Energía y} Recursos Minerales

7 al 11 de Octubre de 2007 Oviedo - España

\section{Informaciones:}

Secretaria del Congreso

secretaria@congresoenerminas.es

http://www.congresoenerminas.es/index.htm

\section{Congreso Nacional de Arqueología Argentina}

8 de 12 de Octubre de 2007

San Salvador de Jujuy - Argentina

Universidad Nacional de Jujuy cnaajujuy@imagine.com.ar ealfaro@inbial.unju.edu.ar http://www.naya.org.ar/eventos/16cnaa.htm

9a Conferencia sobre Calidad y Utilización de la

\section{Electricidad}

9 al 11 de octubre de 2007

Barcelona - España

http://www.epqu2007.com/ CIMNE: epqu07@cimne.upc.edu

CITCEA-UPC: begona mediano@citcea.upc.edu

Mireia.berenguer@citcea.upc.edu

\begin{tabular}{c}
\hline $11^{\text {a }}$ Conferencia Internacional IEEE sobre Visión \\
Artificial, ICCV 2007 \\
14 al 21 de Octubre de 2007 \\
Rio de Janeiro - Brasil \\
Siome Klein Goldenstein \\
UNICAMP, Brasil \\
siome@ic.unicamp.br http://iccv2007.rutgers.edu
\end{tabular}

$3^{\mathrm{er}}$. Simposio IFAC sobre Sistemas, Estructuras y Control

17 al 19 de Octubre de 2007 Foz do Iguazú - Brasil

Secretaria IFAC SSSC07, Univ. Fed.I do Rio Grande do Sul sssc07@ece.ufrgs.br

http://sssc07.ece.ufrgs.br/index.php?link=cont

IV Conferencia Panamericana sobre Ensayos NoDestructivos

22 al 26 de Octubre de 2007

Buenos Aire - Argentina

Asociación Argentina Ensayos No Destructivos y Estructurales info@aaende.org.ar

httr-//www aaende org ar/corende 2006/pre

6 corende e/6corende e.htm

$3^{\text {a }}$ Conferencia Mundial Muestreo y Mezclado (WCSB3)

23 al 25 de Octubre de 2007 Porto Alegre - Brasil

Mining Engineering Department, UFRGS jfelipe@ufrgs.br

http://www.lapes.ufrgs.br/conference/1024/index.html

\section{Noviembre 2007}

\section{Sexto Congreso Iberoamericano de} Ingeniería de Alimentos CIBIA VI

5 al 8 de Noviembre de 2007 Ambato - Ecuador

Universidad Técnica de Ambato fcial@uta.edu.ec http://www.uta.edu.ec/cibiavi/ index.php?option $=$ com frontpage $\&$ temid $=13$

\section{$20^{\circ}$ Congreso y Feria Mundial de Energía}

11 al 15 de Noviembre de 2007 Roma - Italia

Organizing Secretariat, c/o MicroMegas Comunicazione organisingsecretariat@rome2007.it papersandposters@rome2007.it http://www.rome2007.it/home/home.asp 